\title{
Optimized Handover Algorithm Based on Stackelberg Games in CBTC Systems for Urban Rail Transit
}

\author{
Zhang Yanpeng ${ }^{1, *}$, Dang Jianwu ${ }^{1}$, Liu Xiaojuan² and Yang Fan ${ }^{3}$ \\ ${ }^{1}$ School of Automation \& Electrical Engineering, Lanzhou Jiaotong University, Lanzhou, 730070- China \\ ${ }^{2}$ School of Electronics \& Information Engineering, Lanzhou Jiaotong University, Lanzhou, 730070- China \\ ${ }^{3}$ Laboratory LE2I, University of Burgundy, Dijon, 21078-France
}

Received 15 January 2014; Accepted 25 July 2014

\begin{abstract}
Communications Based Train Control (CBTC) systems have become indispensable for train operation to improve the safety and efficiency of train running to the utmost extent at present. In this paper, the problem of train handover in wireless LAN of CBTC systems for urban rail transit was taken into consideration, which was formulated as a market competition with Stackelberg games and cooperative diversity to obtain the reasonable resource allocation for trains. Under the environment of continuous train-to-wayside communications with high reliability, the successful train handover probability was analyzed in detail. Firstly, the relay triggering during handover was introduced, and the price strategies were determined in terms of the bandwidth requested by trains. Then, the utility function of train, based on price and revenue, was designed, and the existence of Nash equilibrium was proved. To achieve the optimal bandwidth strategies of train and network revenue, a distributed iterative learning scheme was proposed to figure out Nash equilibrium. Finally, the train handover process was further analyzed. The results of our study showed that the proposed strategy can motivate relay nodes to participate in cooperation for reasonable resource allocation of networks, the train handover time was less than $50 \mathrm{~ms}$ and the successful train handover probability was remarkably higher than that of the traditional handover of RSS (Received Signal Strength).
\end{abstract}

Keywords: Urban Rail Transit, Communications Based Train Control (CBTC), Wireless LAN, Handover, Stackelberg Game

\section{Introduction}

With the rapid development of information technologies, train control has become increasingly dependent on communications technologies, which has evolved into CBTC (Communications Based Train Control) from traditional TBTC (Track circuit Based Train Control) [1]. CBTC systems for urban rail transit can ensure safe train running, shorten headway time interval and enhance transport capacity to a great extent, on which much attention and application has been focused all over the world. Moreover, CBTC is the perfect type of ATC (Automatic Train Control). In IEEE Std 1474.1, CBTC has been defined as "A continuous ATC system utilizing high-resolution train location determination, independent of track circuits; continuous, high capacity, bidirectional train-to-wayside data communications; and train-borne and wayside processors capable of implementing vital functions" [2]. Obviously, TWC (Train to Wayside Communications) has been one of the key technologies in CBTC systems, of which the high performance is needed to ensure the high reliability and validity of bidirectional data communications when a train is rapidly running on railway lines.

In CBTC systems for urban rail transit, WLAN (Wireless Local Area Network) has been usually adopted to realize the bidirectional data communications between carborne

*E-mail address: lanzhouzyp@126.com

ISSN: 1791-2377 @ 2014 Kavala Institute of Technology. All rights reserved. equipment and ground equipment. For continuous TWC, a train will frequently handover from one AP (Access Point) to next AP, which probably causes the interruption of data transmission in TWC channel. Thus, the issue of handover has become one of the major factors in improving the performance of CBTC systems. Nowadays, the IEEE 802.11 series of standards are widely used in WLAN of CBTC systems for implementing continuous TWC. However, these standards are not intended to be applicable to the frequently rapid moving scenario. When a CBTC-equipped train is running rapidly from one $\mathrm{AP}$ to next $\mathrm{AP}$, handover will take place, which can be divided into three separate phases: scanning, authentication, and association. The experimental and practical testing results show that handover latency is the most crucial factor in realizing the TWC of CBTC systems using WLAN, and the total number of package loss caused by handover is much more than that in normal wireless data transmission [3].

Some scholars at home and abroad in [4-10] have done a great deal of research on handover in wireless communication networks, which mainly analyzed the universal Petri nets model, interruption interval, reliability etc.. The above methods can provide innovative ideas for improving handover algorithm. However, most of the research focused on how to reduce the latency time of a certain phase during handover in WLAN, and scarcely took into account an important point, namely, handover decisionmaking. The traditional algorithm of handover decisionmaking considers only the RSS (Received Signal Strength) 
when deciding handover. Nevertheless, when CBTCequipped trains are rapidly running on railway lines, large scale fading and small scale fading will come into being, which can result in frequent train handover between adjacent APs, produce ping-pong effect and waste communications resources at the same time.

In this paper, we employ a seller/buyer or leader/follower (Stackelberg) game to jointly consider the benefits of trains and relay nodes for continuous communications with high reliability. When a train is rapidly running through two continuous APs, we have taken into consideration many factors, involving bandwidth resources of WLAN, bandwidth requested by trains, cooperative diversity, parameter setting etc., to specifically analyze the train handover process. Then, a mathematical model based on game theory has been established to describe the train handover process and the parameters of price and revenue have been introduced into APs, namely, $(\rho, \theta)$. Furthermore, an iterative learning scheme for mobile nodes has been provided to update node strategies for reasonable resources allocation of network, which can exert the maximum utility of all players in the network and achieve the maximum network revenue. From the above research, the successful train handover probability has been further analyzed and compared by way of illustration.

The rest of this paper is organized as follows: Section 2 describes the handover process and system model in CBTC systems. Section 3 proposes a Stackelberg game model and the relevant algorithm description. Section 4 presents the simulation results. Conclusions are stated in Section 5.

\section{Handover Process and System Model}

CBTC systems for urban rail transit mainly consist of ATS (Automatic Train Supervision), ZC (Zone Controller), CC (Carborne Controller), DCS (Data Communications Subsystem) etc. [11], among which the mutual relationship in data transmission is shown in Fig. 1.

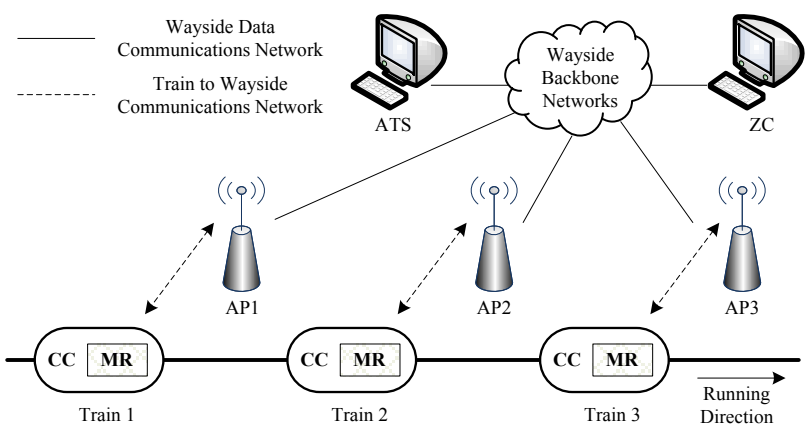

Fig. 1. Information transmission in CBTC systems

ATS is responsible for implementing functions such as identifying, tracking and displaying train information, providing manual and automatic route setting capabilities, and regulating train movements to maintain operating schedules. ZC consists of vital processor-based controllers installed at wayside locations. Each $\mathrm{ZC}$ interfaces to the vehicle $C C$ via the DCS. The function of $Z C$ is to ensure the safe operation of trains using a moving block concept of CBTC. DCS provides bidirectional, secure data communications between CBTC components using UDP/IP protocol. DCS is based on open industrial standards: the wire communication portion adopts Ethernet standards, and the wireless communication portion adopts advanced WLAN technology (IEEE $802.11 \mathrm{~g}$ standards). The mature equipment is used to the greatest extent in CBTC systems. The bottleneck bandwidth of DCS mostly focuses on the bidirectional TWC, that is to say, the wireless link between MR (Mobile Radio) and AP.

To get a simple system model, we consider the specific handover scenario, where a train is rapidly running through two adjacent AP1 and AP2. Suppose there are two mobile nodes in the scenario: a train and some mobile wayside equipment, respectively expressed as $i$ and $j$. The detailed system model of train handover process is shown in Fig. 2.

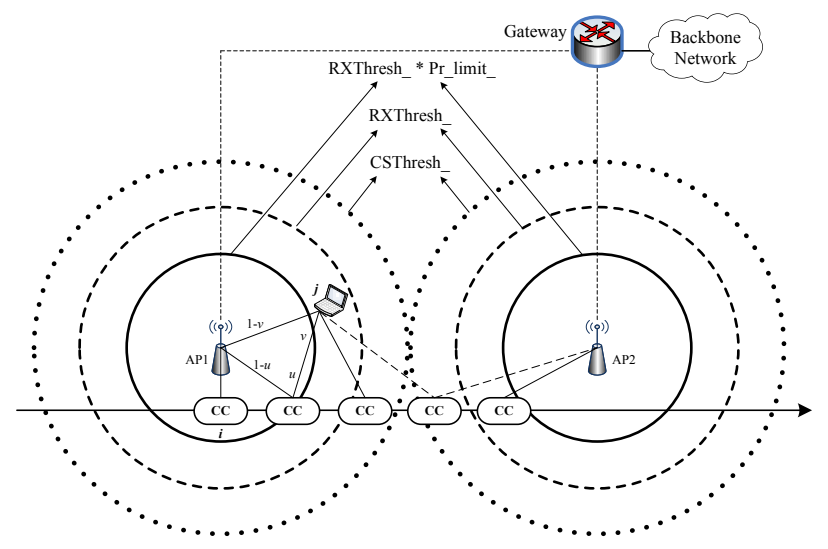

Fig. 2. System model of handover process for a train

As defined in [12], the coverage of an AP can be divided into three levels of hierarchy: CSThresh_, RXThresh_ and RXThresh_*Pr_limit_. The generation of Link Going Down (LGD) is done using the power lever of the received packets. To configure the power level threshold, a coefficient Pr_limit_is used to set this threshold. CSThresh_defines the minimum power lever to sense a packet and switch the MAC from idle to busy (Carrier Sense). RXThresh_defines the minimum power threshold to receive packets without error. RXThresh_*Pr_limit_defines the minimum power threshold before sending LGD event, and Pr_limit_ $>=1$. The higher the coefficient Pr limit, the sooner the event will be generated.

From the above principle, the handover process is carried out in three phases after the initialization of the proposed algorithm when the $(\rho, \theta)$ values are announced by the network. The first phase includes the RXThresh_level, where the train $i$ is running away from AP1 after some time $t$. Meanwhile, the RSS value of RXThresh_*Pr_limit_level received by the train decreases gradually, which will trigger the procedure of data relay. As a result, the node $j$ updates the $(\rho, \theta)$ value, and the parameter $\theta$ denotes the increased incentive for the relaying node $j$.

The second phase takes place when the RSS becomes the minimum within the RXThresh_level and the probability of packet loss between the train $\bar{i}$ and AP1 increases. At the time, it is difficult for the train $i$ to transmit its data through AP1. So, the node $j$ updates the $(\rho, \theta)$ value again. Once the train runs into the CSThresh_level, the node $j$ starts relaying most of the data stream of the train $i$.

The final phase starts when the RSS is approximately equal to zero within the CSThresh level. Meanwhile, the link between the train $i$ and AP1 cuts off. The crucial phase lasts until the train $i$ ultimately detects the CSThresh_level 
of AP2. When the train $i$ runs into the minimum of the RXThresh level, the train $i$ sends a router solicitation message to AP2. If the train $i$ associates with AP2, the train $i$ will terminate the link with the node $j$ and receive the acknowledgement of route diversion through AP2.

In the course of the above handover in wireless LAN, we assume that both transmission bandwidth of $i$ and $j$ are equal to $\omega \mathrm{MHz}$, and the node $j$ is willing to use the $\omega_{j}$ fraction of its channel to relay the $\omega_{j}$ fraction of data stream for the node $i\left(0 \leq \omega_{j} \leq \omega\right)$. As cooperative transport protocols, AF (Amplify and Forward) has been adopted to achieve the cooperative diversity gain [13]. $P_{i}$ and $P_{j}$ are respectively the transmission power of the nodes $i$ and $j . \gamma_{i, \alpha}, \gamma_{i, j}$ and $\gamma_{j, \alpha}$ denote the respective SNR (Signal Noise Ratio) of different three routes (between $i, j$ and AP1). Therefore, the received SNR of the whole system can be expressed as $\gamma_{i, \alpha}{ }^{\text {relay }}=\gamma_{i, \alpha}$ $+\gamma_{i, j, \alpha}$, and $\gamma_{i, j, \alpha}=\gamma_{i, j} \gamma_{j, \alpha} /\left(1+\gamma_{i, j}+\gamma_{j, \alpha}\right)$.

\section{Stackelberg game model and algorithm description}

The train handover process can be described as a Stackelberg game model, in which there are two players, namely, the leader $j$ and the follower $i$. Both players aim at the maximum utility of self in the network and the follower $i$ will choose its strategies according to the leader $j$ 's strategies. Therefore, the leader $j$ will set the price strategies on the base of the total bandwidth requirements of all nodes to affect the bandwidth requirements of other nodes for the maximum utility of self in the network. The follower $i$ will adjust the bandwidth requirement strategies of self in accordance with price and self utility in the network.

Suppose the node set of the followers in the network is $\mathrm{N}=\{1,2, \ldots, n\}$, and $i \mathrm{~N}$. The strategy of the node $i$ is the purchased cooperative bandwidth $\left(\omega_{i} \mathrm{MHz}\right)$ from the leader $j$. So the price strategy of the leader $j$ can be defined as

$$
c(\omega)=y\left(\sum_{i=1}^{N} \omega_{i}\right)^{\tau}
$$

where $\omega=\left\{\omega_{1}, \ldots, \omega_{n}\right\}$ is the strategy combination of all nodes, $y$ and $\tau$ are non-negative constants, $\tau \geq 1$, so that the price function $c(\omega)$ is convex [14].

\subsection{Utility Function}

The utility can reflect the satisfactory extent of the strategies chosen by players in the game, and be specifically described through utility function. Each node in the network expects to achieve much higher throughput $T h$ with lesser transmission power $P$. The above problem can be compromised by the energy efficiency function $u$, namely, $u=T h / P \mathrm{bit} /(\mathrm{s} \cdot \mathrm{W})=$ $T h / P$ bit/J.

Suppose the nodes transmit their data with the frame of $\mathrm{M}$ bits at the rate of $\mathrm{R}$ bit/s under the bandwidth $\omega$, and the useful data is $\mathrm{L}(\mathrm{L}<\mathrm{M})$ bits. Then, the throughput can be expressed as $T h=\mathrm{R} \cdot f(\gamma) \cdot \mathrm{L} / \mathrm{M}$, where $f(\gamma)=\left[1-2 \mathrm{BER}_{2 \mathrm{FSK}}(\gamma)\right]^{\mathrm{M}}$ is the approximation function of the probability of a successfully received frame, $\operatorname{BER}_{2 \mathrm{FSK}}(\gamma)=\mathrm{e}^{-\gamma / 2}$, and $\mathrm{R}=\eta \omega(\eta$ denotes the utilization of bandwidth) $[15,16]$. If $\eta_{2 \mathrm{FSK}}=1$ $\mathrm{bit} / \mathrm{s} / \mathrm{Hz}$, the throughput can be expressed as $T h=\mathrm{R} \cdot f(\gamma) \cdot \mathrm{L} / \mathrm{M}=\omega \cdot f(\gamma) \cdot \mathrm{L} / \mathrm{M}$.

From the above conditions, the utility function of the node $i\left(\begin{array}{ll}i & \mathrm{~N}\end{array}\right)$ can be defined as

$$
U_{i}=\left(T h_{i, \alpha}\left(P_{i}, \omega-\omega_{i}\right)+T h_{i, \alpha}^{\text {Relay }}\left(P_{i}, \omega_{i}\right)\right) / P_{i}-c \cdot \omega_{i}
$$

where $c \cdot \omega_{i}$ is the payoff of cooperative bandwidth occupied by the node $i$.

\subsection{Nash Equilibrium}

Nash equilibrium is the solution of non-cooperative game. When the Nash equilibrium is achieved, the utility function of each player in the non-cooperative game reaches the maximum, and the unilateral changes of the players' strategies will not increase their revenue [17]. However, every non-cooperative game has not the Nash equilibrium. We should prove the existence of Nash equilibrium in the game.

Theorem 1: If game $\mathbf{G}=\left[\mathrm{N},\left\{W_{i}\right\},\left\{U_{i}(\cdot)\right\}\right]$, where $W_{i}=$ $\left\{0, \omega_{1}, \omega_{2}, \ldots, \omega_{n}\right\}\left(0 \leq \omega_{1} \leq \omega_{2} \leq \ldots \leq \omega_{\mathrm{n}}\right)$ is the strategy space of the players, and $U_{i}(\cdot)$ is its utility function, then the Nash equilibrium exists for " $i \mathrm{~N}$.

Proof: Clearly, the strategy space of bandwidth for any node $i$ is the bounded closed set in Eucilidean spaces, and the utility function $U_{i}$ is continuous in its strategy space. Now, we prove the utility function $U_{i}$ is concave.

Substituting (1) into (2), after some manipulations, we can conclude that

$U_{i}=\frac{L \omega}{M P_{i}} f\left(\gamma_{i, \alpha}\right)+\frac{L \omega_{i}}{M P_{i}} \Delta f\left(\gamma_{i, \alpha}^{\text {Relay }}, \gamma_{i, \alpha}\right)-\omega_{i}\left(y\left(\sum_{j=1}^{N} \omega_{j}\right)^{\tau}\right.$

where $\Delta f\left(\gamma_{i, \alpha}^{\text {Relay }}, \gamma_{i, \alpha}\right)=f\left(\gamma_{i, \alpha}^{\text {Relay }}\right)-f\left(\gamma_{i, \alpha}\right)$ denotes the SNR gain from cooperative diversity for the node $i$.

For any node $i$, taking the first-order derivative of the utility function $U_{i}$ with respect to $\omega_{i}$, we have

$$
\frac{\partial U_{i}}{\partial \omega_{i}}=\frac{L}{M P_{i}} \Delta f\left(\gamma_{i, \alpha}^{\text {Relay }}, \gamma_{i, \alpha}\right)-y\left(\left(\sum_{j=1}^{N} \omega_{j}\right)^{\tau}+\tau \omega_{i}\left(\sum_{j=1}^{N} \omega_{j}\right)^{\tau-1}\right)
$$

Similarly, for any node $i$, taking the second-order derivative of the utility function $U_{i}$ with respect to $\omega_{i}$ and $\omega_{j}$, we obtain

$$
\frac{\partial^{2} U_{i}}{\partial \omega_{i}^{2}}=-\left[2 y \tau\left(\sum_{j=1}^{N} \omega_{j}\right)^{\tau-1}+\omega_{i} y \tau(\tau-1)\left(\sum_{j=1}^{N} \omega_{j}\right)^{\tau-2}\right]
$$

$\frac{\partial^{2} U_{i}}{\partial \omega_{i} \partial \omega_{j}}=-\left[y \tau\left(\sum_{j=1}^{N} \omega_{j}\right)^{\tau-1}+\omega_{i} y \tau(\tau-1)\left(\sum_{j=1}^{N} \omega_{j}\right)^{\tau-2}\right]$

Since $\tau \geq 1$ and $y>0, \partial^{2} U_{i} / \partial \omega_{i}^{2}<0$ and $\partial^{2} U_{i} / \partial \omega_{i} \partial \omega_{j}<0$. Thus, the utility function $U_{i}$ for the node $i$ is strictly concave and joint. Therefore, the Nash equilibrium of game $\mathbf{G}$ exists.

\subsection{Solution of Stackelberg Game Problems}

The problems of Stackelberg game usually use backward induction to resolve. However, backward induction is a solution with complete information, that is to say, each player in the game must know about the relevant information of other players, which is quite difficult to carry out in wireless network systems $[18,19]$. In the paper, a distributed iterative learning scheme for mobile nodes has been provided to get the subgame perfect Nash equilibrium in Stackelberg games, in which each player should only know about a little partial information in the network.

We assume that $\mathrm{N}$ is the set of mobile nodes in wireless network, and each mobile node has a set with finite actions. Now, we consider a scenario of two mobile nodes $(i, j)$, in which $\mathrm{A} 1$ and $\mathrm{A} 2$ are their respective sets of finite actions. 
For each player $(i, j \quad \mathrm{~N}), a_{i} \quad \mathrm{~A}_{i}$ denotes the action chosen by the node $i$. The learning rates $\lambda_{i, t}$ and $v_{i, t}$ are respectively assigned for the strategy update function $x_{i, t+1}\left(a_{i}\right)$ and estimated payoff function $\hat{u}_{i, t+1}\left(a_{i}\right)$, as shown in (7). $\Gamma_{a i, t=a i}$ is an indicator function for the chosen action $a_{i}$ at time $t$ and $U_{i, t}$ is the observed utility function for any node $i[20]$.

$\left\{\begin{array}{l}x_{i, t+1}\left(a_{i}\right)=x_{i, t}\left(a_{i}\right)+\lambda_{i, t} U_{i, t}\left(\Gamma_{a i, t=a i}-x_{i, t}\left(a_{i}\right)\right) \\ \hat{u}_{i, t+1}\left(a_{i}\right)=\hat{u}_{i, t}\left(a_{i}\right)+v_{i, t} \Gamma_{a i, t=a i}\left(U_{i, t}-\hat{u}_{i, t}\right)\end{array}\right.$

In the wireless network, $U_{i, t}$ is regarded as the strategy chosen by $x_{i, t}\left(a_{i}\right)$ at time $t$, and $a_{i}$ is the chosen action. The strategy function $x_{i, t+l}\left(a_{i}\right)$ is updated through (7). Similarly, the estimated utility function $\hat{u}_{i, t+l}\left(a_{i}\right)$ is also updated through the same procedure. The utility functions of $i$ and $j$ are respectively formulated as (8) and (9). $f(\cdot)$ is the efficiency function in [21], and the nodes $i$ and $j$ use (8) and (9) to optimize the utility of themselves.

$$
\begin{gathered}
\text { Node 1: } \max U_{i}\left(P_{i}, u\right) \equiv \\
\max \left(\frac{1}{P_{i}}-\rho\right)\left[(1-\rho) f\left(\gamma_{i, \alpha}\left(P_{i}\right)\right)+u f\left(\gamma_{i, j}\left(P_{i}\right)\right)\right] \\
\text { s.t. }\left\{\begin{array}{c}
0 \leq P_{i} \leq P_{\max }, 0 \leq u \leq 1, \\
u f\left(\gamma_{n i, j}\left(P_{i}\right)\right) \leq v f\left(\gamma_{j, \alpha}\left(P_{j}\right)\right)
\end{array}\right.
\end{gathered}
$$

Node 2: $\max U_{j}\left(P_{j}, v\right) \equiv$

$$
\begin{aligned}
& \max \left[(1-v)\left(\frac{1}{P_{i}}-\rho\right)+k(\theta-\rho)\right] f\left(\gamma_{j \alpha}\left(P_{j}\right)\right) \\
& \text { s.t. }\left\{\begin{array}{c}
0 \leq P_{j} \leq P_{\max }, 0 \leq v \leq 1, \\
l f\left(\gamma_{i, j}\left(P_{i}\right)\right) \geq v f\left(\gamma_{j, \alpha}\left(P_{j}\right)\right)
\end{array}\right.
\end{aligned}
$$

For every pair $(\rho, \theta)$, the two nodes mutually compete to maximize their utility of self through (8) and (9) according to the different strategies in (7). Once the point of Nash equilibrium is achieved, the network makes use of the equilibrium values $\left(P_{i}{ }^{*}, u^{*}\right)$ and $\left(P_{j}{ }^{*}, v^{*}\right)$ to calculate the network revenue $R_{\text {net }}$ for different $(\rho, \theta)$ values through (10).

$$
R_{\text {net }}\left[\left(P_{i}^{*}, u^{*}\right),\left(P_{j}^{*}, v^{*}\right)\right]=\sum \lambda\left(T h_{i}\right)-\sum \lambda\left(T h_{j}\right)
$$

The whole process of the iterative learning scheme for mobile nodes can be described as follows:

$a$. Initialize the network parameters, such as the $(\rho, \theta)$ values, power and bandwidth of the nodes, price strategies and so on.

$b$. Update the $(\rho, \theta)$ values during the different phases after the relay triggering. According to (7), the nodes will adjust their bandwidth requirements until their utility comes to the maximum and Nash equilibrium for all nodes comes into being.

$c$. If the utility of all nodes comes to the maximum at the time, the iteration stops. Otherwise, at the next time $t+1$, the step will return to step $a$ for continuous iteration.

\section{Simulation and result analysis}

\subsection{Parameter Setting}

We consider a train handover process in WLAN environment, where a train is running at the constant speed of $100 \mathrm{~km} / \mathrm{h}$ from AP1 to AP2. The specific parameters of train handover process can be set as Tab. 1 .

\begin{tabular}{l|l} 
Table 1. Simulation parameters of train handover process \\
\hline Parameter & Value \\
\hline Technical standards & IEEE $802.11 \mathrm{~g}$ \\
ISM band & $2.4 \mathrm{GHz}$ \\
Radius of an AP & $150 \mathrm{~m}$ \\
Distance between AP1 and AP2 & $200 \mathrm{~m}$ \\
Distance between AP1 and the node $j$ & $50 \mathrm{~m}$ \\
Transmission power of AP & $20 \mathrm{dBm}$ \\
Modulation scheme & OFDM/CCK/DSSS \\
Bandwidth & $22 \mathrm{MHz}$ \\
Data transmission rate & Max: $54 \mathrm{Mbps}$ \\
Wireless channel model & Hata model \\
Frame & $10 \mathrm{~ms}$ \\
Iterative times & 200 \\
Learning factor & 2 \\
\hline
\end{tabular}

In addition, we set $\mathrm{M}=80, \mathrm{~L}=64, \tau=2, y=10^{-10}$ in the simulation.

\subsection{Analysis of simulation results}

When the price strategies are advertised in the wireless LAN, the competition process based on non-cooperative game among the nodes has been analyzed in detail. Then, the probability of successful train handover has been further analyzed. The specific competition process, such as bandwidth strategies, price strategies, effect of price on utility functions, network revenue and so on, has been shown in Fig. 3, Fig. 4 and Fig. 5.

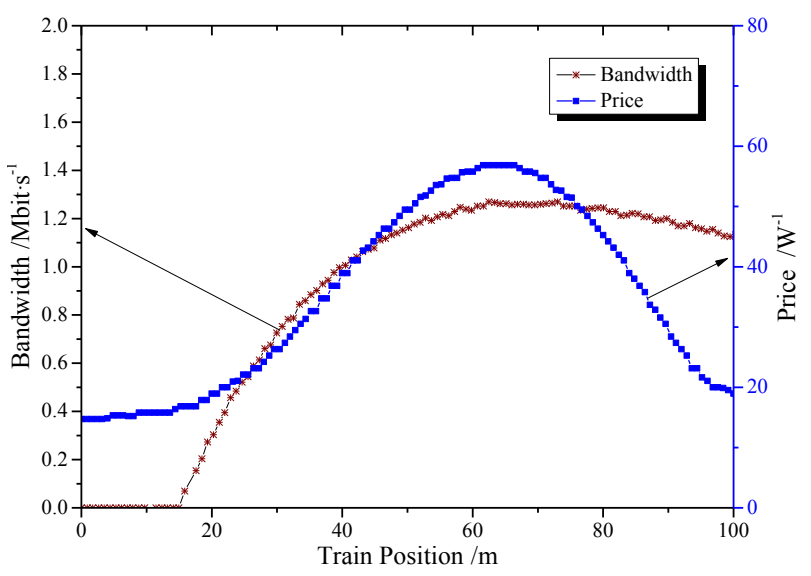

Fig. 3. Optimal purchase quantity of bandwidth and price strategies for the train

In Fig. 3, the variation curves of bandwidth strategies and price strategies have been given when a train is running at the constant speed of $100 \mathrm{~km} / \mathrm{h}$ from AP1 to AP2. During the course of train running from $0 \mathrm{~m}$ to $50 \mathrm{~m}$, the bandwidth requested by the train from the relaying node is ever increasing, so the resource price of the relaying node is also boosted continuously. With the rapid train running, the bandwidth requested by the train from the relaying node begins to decrease slowly and the relaying node also reduces the price to attract the train for purchasing the bandwidth. In the simulation, the train becomes triggering the relay in the 
position of $15.62 \mathrm{~m}$ and starts purchasing the bandwidth. From now on, the bandwidth price is increasingly boosted. In the position of $61.28 \mathrm{~m}$, the bandwidth demand is maximum and the bandwidth price is also highest. Then, the train is gradually running away from the relaying node. Meanwhile, the bandwidth demand of the train become decreasing and the bandwidth price is also reduced, which will last until the connection between the train and AP2 establishes.

In Fig. 4, the variation curve of train utility with respect to price is shown. From the curve, we can clearly make out the utility function of train is concave with respect to price. When the price becomes boosted from 16.8, the train utility increases with the price. While the price is at 58.6, the train utility becomes maximum. As a result of increased price, the network load decreases sharply and the network revenue begins to fall.

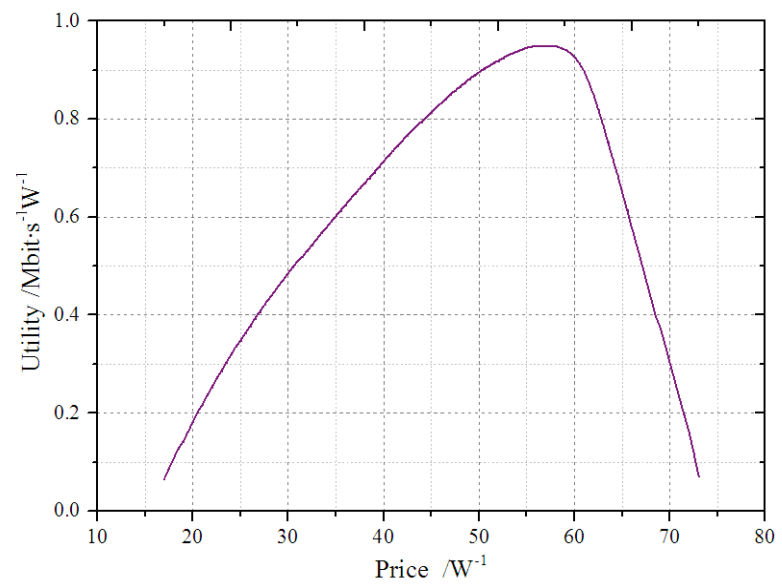

Fig. 4. Curve of train utility

In Fig. 5, the variation curve of train revenue with respect to iterative times is presented. We can clear about the train revenue is increasing with the gradually increased bandwidth requested by the train from the relaying node, tends to convergence and stabilize at the point of Nash equilibrium.

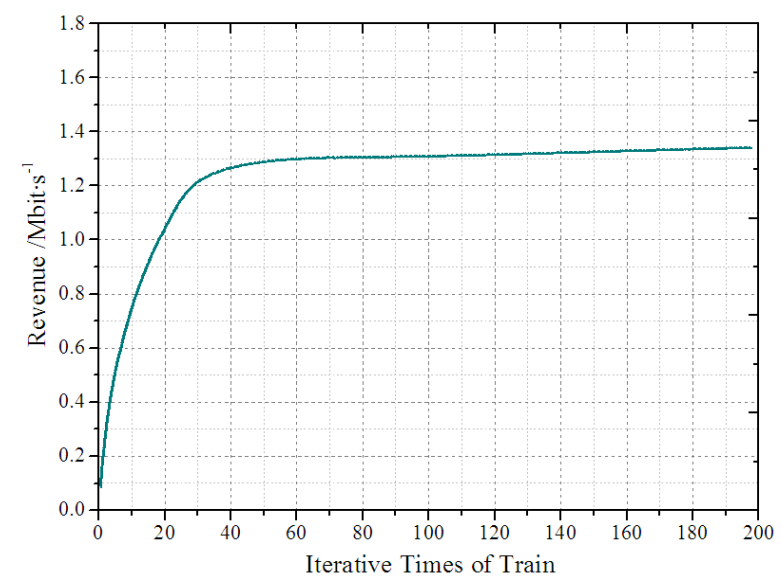

Fig. 5. Relation between network revenue and iterative times of train

From the handover process, when rapid running in the area covered by AP1, a train will keep connecting with AP1. Then, the train runs into the area covered together by AP1 and AP2. Meanwhile, the connection between the train and AP1 is still maintained. Compared with the traditional handover algorithm of RSS, the proposed handover algorithm based on Stackelberg game has the advantages of advanced connection establishment. For example, the train will connect AP2 ahead, and will not search for a new AP to connect when the RSS of AP1 is the least.

The relation between handover time and successful train handover probability is shown in Fig. 6. Between zero and $3575 \mathrm{~ms}$ during the simulation, the two curves don't separate from each other, and the successful handover probability of two methods are identical, that is to say, equal to zero. Between $3576 \mathrm{~ms}$ and $3612 \mathrm{~ms}$, the successful handover probability of the proposed method in the paper becomes elevated quickly and tends to a stable value, approximate to $99.812 \%$, which meets the demand that the successful handover probability is greater than $99.5 \%$. Meanwhile, the dynamic handover time is less than $36 \mathrm{~ms}$. Between $3588 \mathrm{~ms}$ and $3692 \mathrm{~ms}$, the successful handover probability of traditional RSS handover becomes heightened rapidly and tends to a stable value. The dynamic handover time of RSS is approximate to $104 \mathrm{~ms}$. From the simulation results, we conclude that the proposed handover algorithm based on Stackelberg game distinctly excels the traditional handover algorithm of RSS, which can establish the connection with the coming AP ahead, satisfy the technical requirements that the dynamic handover time is less than $50 \mathrm{~ms}$ and the whole handover time is less than $100 \mathrm{~ms}$ [22].

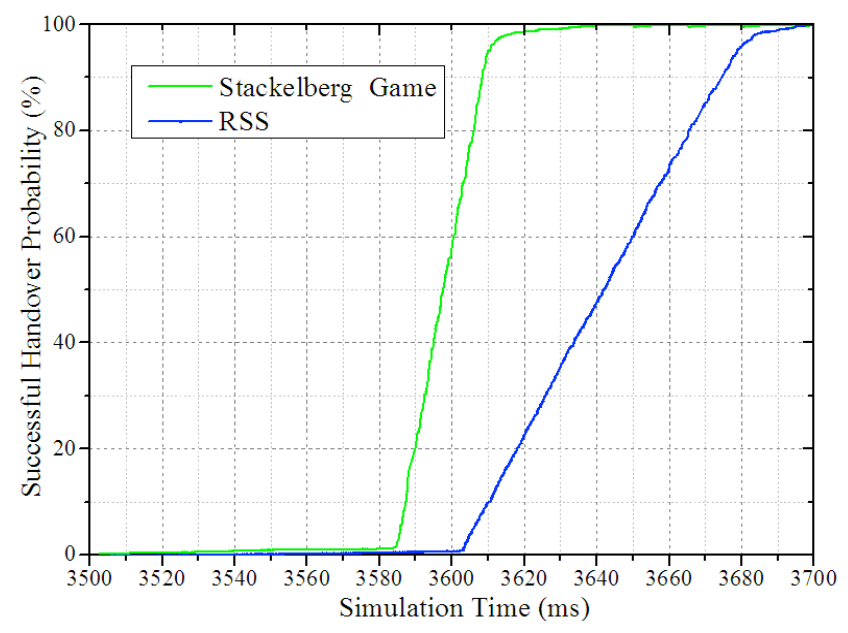

Fig. 6. Successful train handover probability with different strategies

\section{Conclusions}

We have proposed a train handover algorithm based on game theory for CBTC systems in urban rail transit, which has taken into account the interests of all nodes in wireless LAN and has adopted Stackelberg games to analyze the mutual relation between train and relaying node. On the base of the determination of price strategies for mobile nodes, a non-cooperative game comes into being and the point of Nash equilibrium of non-cooperative game is proved existing. Then, a distributed iterative learning scheme for mobile nodes has been provided to achieve the optimal price strategies and maximum utility, which has realized the resource allocation with high efficiency in wireless LAN. Given the relevant parameters, the train handover process has been further analyzed. The simulation results have shown the train handover time can adequately satisfy the technical requirements of less than $100 \mathrm{~ms}$ for CBTC systems in urban rail transit, and the successful handover 
probability has also reached the needs of safe data transmission.

\section{Acknowledgment}

The study thanks the projects and their team members supported by "Natural Science Foundation for Young
Scientists of Gansu Province, China (Grant No. 1308RJYA057)", "Science and Technology Support Plan of Lanzhou Jiaotong University, China (Grant No. ZC2014004)", and "Natural Science Foundation for Young Scientists of Lanzhou Jiaotong University, China (Grant No. 2012036)".

\section{References}

1. Liu Xiaojuan, Zhang Yanpeng, Tang Zian.: Intelligent Control Systems for Urban Rail Transit, China Railway Press, Beijing, 2008

2. IEEE Standard Association. IEEE Standard for User Interface Requirements in Communications-Based Train Control (CBTC) Systems, New York: The Institute of Electrical and Electronics Engineers, Inc, 2003.

3. JIANG Hai-lin, BU Bing, GAO Chun-hai, et al, "Location-based Handover Scheme for Wireless LAN Networks in CBTC System", Journal of the China Railway Society, 33(10), 2011, pp. 51-56.

4. CAO Yuan, MA Lianchuan, ZHANG Yuzhuo, et al, "Comparision of Handover Success Rates between LTE-R and GSM-R", China Railway Science, 34(6), 2013, pp.117-123.

5. CAO Yuan, NIU Ru, TANG Tao, MU Jiancheng, "Analysis of HandoverModel Based on Stochastic Petri Net", Journal of the China Railway Society, 31(4), 2009, pp.104-107.

6. LIU Hong-jie,CHEN Li-jie, "Research on Time of Communication Interruption in Safe Positioning of CBTC Train", Journal of the China Railway Society, 34(6), 2012, pp.40-45.

7. CHEN Lijie,TANG Tao,LU Jidong, "Analysis on the Interruption Time for CBTC Handover", China Railway Science, 31(5), 2010, pp.125-129.

8. Tran Cong Hung, Bui Tran Thanh Thao and Nguyen Thien Binh, "Handover in Wireless Mesh Network". Journal of Communication and Computer, 7(8), 2010, pp.24-30.

9. Md. Imtiyaz Anwar, Arun Khosla, Neetu Sood, "A Mobility Improvement Handover Scheme for Mobile-WiMAX", International Journal of Computer Applications, 11(3), 2010, pp. 28-31.

10. T. Charles Clancy, "Secure handover in enterprise WLANs: CAPWAP, HOKEY, and IEEE 802.11r", IEEE Wireless Communications, 15(5), 2008, pp. 80-85.

11. IEEE Standard Association. IEEE Recommended Practice for Communications-Based Train Control (CBTC) System Design and Functional Allocations, New York: The Institute of Electrical and Electronics Engineers, Inc, 2008.

12. National Institute of Standards and Technology. The Network Simulator NS-2 NIST add-on Mac 802.11, Maryland: National Institute of Standards and Technology (NIST), 2007.
13. Hanan Al-Tous and Imad Barhumi, "Joint Power and Bandwidth Allocation for Amplify-and-Forward Cooperative Communications Using Stackelberg Game", IEEE Transactions on Vehicular Technology, 62(4), 2013, pp.1678-1691.

14. Dusit Niyato and Ekram Hossain, "Competitive Spectrum Sharing in Cognitive Radio Networks: A Dynamic Game Approach”, IEEE Transactions on Wireless Communications, 7(7), 2008, pp.26512660.

15. Cem U. Saraydar, Narayan B. Mandayam, and David J. Goodman, "Efficient Power Control via Pricing in Wireless Data Networks", IEEE Transactions on Communications, 50(2), 2002, pp.291-303.

16. CONG Li,ZHANG Hai-lin,LIU Yi, et al, "Particle Swarm Optimized Game Theory for Resource Allocation in Cooperative Networks", Journal of Jilin University (Engineering and Technology Edition), 42(1), 2012, pp. 207-212.

17. JIANG Yong,CHEN Shan-zhi,HU Bo, "Stackelberg Games-Based Distributed Algorithm of Pricing And Resource Allocation in Heterogeneous Wireless Networks", Journal of communications, 34(1), 2013, pp.61-68.

18. Beibei Wang, Zhu Han, K.J. Ray Liu, "Distributed Relay Selection and Power Control for Multiuser Cooperative Communication Networks Using Stackelberg Game", IEEE Transactions on Mobile Computing, 8(7), 2009, pp.975-990.

19. Dusit Niyato and Ekram Hossain, "Competitive Pricing for Spectrum Sharing in Cognitive Radio Networks: Dynamic Game, Inefficiency of Nash Equilibrium, and Collusion". IEEE Journal on Selected Areas in Communications, 26(1), 2008, pp.192-202.

20. E. J. Collins and David S. Leslie, "Convergent Multiple-timescales Reinforcement Learning Algorithms in Normal Form Games", The Annals of Applied Probability, 13(4), 2003, pp.1231-1251.

21. Tamer ElBatt and Anthony Ephremides, "Joint Scheduling and Power Control for Wireless Ad Hoc Networks", IEEE Transactions on Wireless Communications, 3(1), 2004, pp. 74-85.

22. Ministry of Housing and Urban-Rural Development of the People's Republic of China (MOHURD). CJ/T 407-2012 Technical Requirements of Communication Based Automatic Train Control System for Urban Rail Transit. Beijing: Standards Press of China, 2013. 